
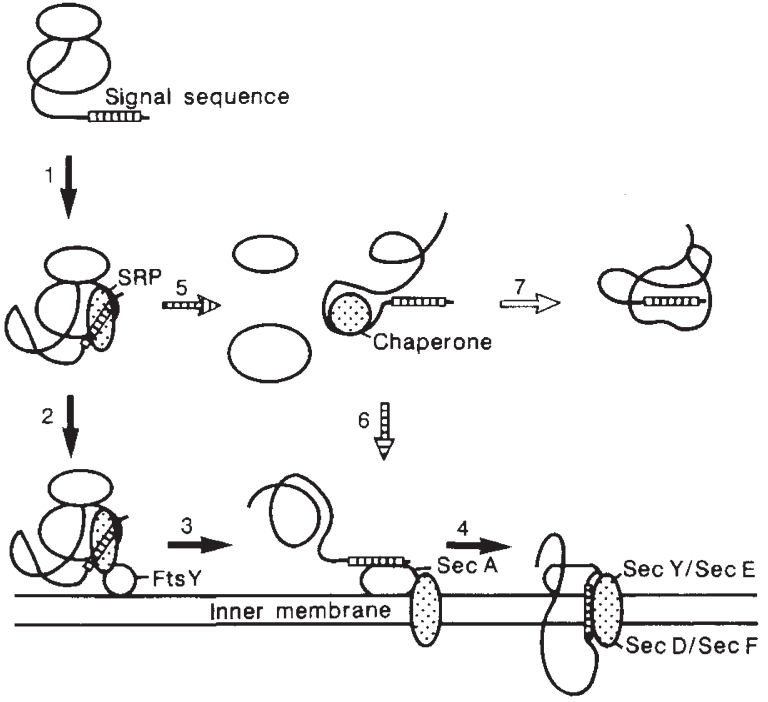

Possible pathways of protein translocation in Escherichia coli. Step 1, the signal sequence has emerged from the ribosome and may bind the signal recognition particle (SRP). Step 2, the complex of nascent polypeptide chain, SRP and ribosome binds to the inner membrane, probably through FtsY. Step 3, the nascent chain is transferred to SecA. Step 4 , transfer of the polypeptide through the membrane, with the involvement of the integral membrane proteins SecY, SecE and, probably, SecD and SecF. Step 5, proteins that have missed their chance of cotranslational targeting may bind a chaperone. Step 6 , they may then be transferred to SecA and converge in the common pathway. Step 7, alternatively they may misfold and not become translocated. Some proteins, such as the M13 procoat, may be directly transferred across the membrane after step 5 , bypassing SecA and the inner membrane proteins $^{17}$. Many of the steps shown as undirectional may in fact be equilibria.

proteins (SecY, SecE, SecD, SecF) $)^{1+}$.

I favour an alternative possibility that would unify the function of SRP in nature. SRP-independent translocation would only serve as a salvage pathway for precursor proteins that have missed their chance of cotranslational targeting by SRP. In this case, the SRP pathway would be the basic one, alleviating constraints that particular folding characteristics of a protein might impose on its translocation. It may be assumed that all signal sequences interact with SRP to form a complex, but with different binding constants. The concentration of the complex would then determine the rate of translocation. Peptide chains with weak signal sequences (like, presumably, that of pre- $\beta$ lactamase) may be completed in a larger proportion before SRP-dependent translocation. They may then be rescued for transport by interaction with chaperones. Alternatively, aberrant folding may make them incompetent for translocation. If the level of functional SRP is lowered, or if its membrane interaction is retarded by excess $\mathbf{P} 48$, proteins with signal sequences of low affinity for SRP may be affected first. Among the latter (besides $\beta$-lactamase) may be some which use the salvage pathway insufficiently and are essential for the viability of the cell. Death would occur before the level of SRP is lowered so far as to exert an effect on exported proteins with stronger signal sequences. Under normal conditions, only a few proteins would use the salvage pathway and need a chaperone (SecB, for example) $)^{15}$. Under unfavourable conditions more proteins are forced into this pathway and heat shock protcins are called on. Adaptation of folding properties of proteins to such conditions may be common in bacteria.

According to this model, the process in eukaryotes differs only quantitatively. The eukaryotic translation mechanism is much slower and there is enough time for the growing polypeptide chain to bind SRP well before its completion. As a consequence, the folding properties of the mature portion of an exported protein will have less influence on the efficiency of translocation than in bacteria. Nevertheless, a salvage pathway may exist even in eukaryotes at least for short polypeptide chains, and a number of proteins can indeed be translocated posttranslationally with the aid of chaperones ${ }^{1 \mathrm{th}}$.

It is now clear that not only are the signals for protein translocation the same in all organisms, but that they are recognized by molecules (P48 or SRP54) that have been highly conserved in evolution. Given that, one may hope that other basic features of the translocation apparatus will also turn out to be universal.

Tom A. Rapoport is in the Central Institute of Molecular Biology, Robert-Rössle-Strasse 10. 1115 Berlin-Buch, Germany.

1. Ribes, V., Römisch, K., Giner, A., Dobberstein, B. \& Tollervey, D. Cell63, 591-600 (1900)

. Poritz, M.A., Bernstein, H. Strub K., Zopf, D. Wilhelm, H. \& Walter P. Science 250, 1111-1117 (1990).

Rapoport. T.A. Trends biochem. Sci. 15, 355-358 (1990).

4. von Heijne, G. Curr. Topics Membr. Trans. 24, 151-179 (1985)

. Römisch, K. et al. Nature 340, 478-482 (1989)

. Bernstein, H.D. et al. Nature 340, 482-486 (1989)

Brown, S. \& Fournier, M.J. J. molec. Biol. 178, 533-550 (1984).

8. Brown, S. J molec Biol 209, 79-90 (1989)

9. Struck, J.C.R. Toschka H.Y Specht, T. \& Erdmann, V.A. Nucl. Acids Res. 16, 7740 (1988)

10. Bernstein, H.D., Rapoport, T.A. \& Walter, P. Cell $\mathbf{5 8}$ 1017-1019 (1989)

11. Randall, L.L., Topping, T.B. \& Hardy, S.J.S. Science 248 860-863 (1990)

12. Hartl, F.-U., Lecker, S., Schiebel, E., Hendrick, J.P. \& Wickner, W. Cell63, 269-279 (1990)

3. Lill, R et al. EMBO J. 8, 961-966 (1989)

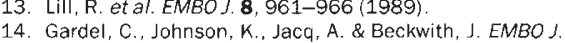
9, 3209-3216 (1990)

15. Kumamoto, C.A. \& Beckwith, J. J. Bacteriol. 163, 267274 (1985).

16. Zimmerman, R. \& Meyer, D.I. Trends biochem. Sci. 11. $512-515$ (1986)

17. Wolfe, P. B., Rice, M. \& Wickner, W. J. biol. Chem. 260. 1836-1841

\section{Shaped charges}

PLASTIC moulding is the simplest of processes. You just melt the polymer and force it into a steel mould of the shape you want. The vast expense of the mould limits you to products which can be sold in identical millions. Daedalus is now changing all this. $\mathrm{He}$ has been inspired by electron-beam thermoplastic recording, in which an electron beam writes a pattern of charge density onto a moving plastic tape, which then passes through a heat-softening chamber. The charged regions repel each other, deforming the tape surface into a pattern of dense ripples which can be read optically. DREADCO engineers are scaling up the process. They are passing continuously extruded plastic tube or rod through a vacuum chamber, and dumping quite ferocious patterns of charge density onto it from a battery of programmed electron guns. On subsequent heating, it expands and deforms dramatically into the shape defined by the imposed charge-pattern.

The new 'thermoelectroforming' process is a computational nightmare. Even simple symmetrical objects like bowls and buckets pose severe challenges, while DREADCO mathematicians are still struggling vainly to calculate the exact pattern of charge needed to deform a length of plastic rod into a complex object like a chair, a computer keyboard or a telephone handset. But traditional plastic-fabrication moulds are so expensive and inflexible that any way of defining a shape in software rather than steel should be worth all the pain of development.

Even better, a thermoelectroformed product can be improved or modified at any time by simple software patching, rather than making new expensive moulds. Instead of being limited to mass designs, plastics manufacturers will be able to tweak and customize their products at will. They will move up-market into specialized niches, indulging their customers with personal flourishes and elegant variations on designs which will themselves be continually optimized and upgraded.

And thermoelectroformed articles will be beautiful. Despite the specific engineering requirements, the overall shape of a plastic object is usually pretty arbitrary. Steelmould technology has encouraged such universally tasteless styling that the very word 'plastic' has become a term of abuse. But thermoelectroforming will produce flowing, organic, integrated shapes, naturally defined and unified by the electrostatic field-equations. Good designers will cease to impose arbitrary styling on such sculptural creations. Instead they will relish the charm and economy of the shapes arising naturally from simple charge distributions. A new elegance and style will infiltrate our graceless age of plastic.

David Jones 